\title{
HOW LONG DOES IT TAKE FOR A GAS TO FILL A POROUS CONTAINER?
}

\author{
CARMEN CORTÁZAR AND MANUEL ELGUETA
}

(Communicated by John Trangenstein)

\begin{abstract}
Let us consider the problem $u_{t}(x, t)=\Delta u^{m}(x, t)$ for $(x, t) \in$ $D \times[0,+\infty), u(x, 0)=u_{0}(x)$ for $x \in D$, and $\left(\partial u^{m} / \partial n\right)(x, t)=h(x, t)$ for $(x, t) \in \partial D \times[0,+\infty)$. Here we assume $D \subset R^{N}, m>1, u_{0} \geq 0$, and $h \geq 0$. It is well known that solutions to this problem have the property of finite speed propagation of the perturbations. By this we mean that if $z$ is an interior point of $D$ and exterior to the support of $u_{0}$, then there exists a time $T(z)>0$ so that $u(z, t)=0$ for $t<T(z)$ and $u(z, t)>0$ for $t>T(z)$.

In this note we give, in an elementary way, an upper bound for $T(z)$ for the case of bounded convex domains and in the case of a half space.
\end{abstract}

\section{INTRODUCTION}

The standard model for the flow of gas through a porous medium stands as follows. Suppose we have a gas in a container that we model as a region $D \subset R^{N}$. Let us denote by $u(x, t)$ the density of the gas on the point $x$ at the instant $t$. Analogously we denote by $p(x, t)$ and $\vec{v}(x, t)$ its pressure and its velocity, respectively.

The law of conservation of mass implies

$$
u_{t}+\operatorname{div}(u \vec{v})=0 \text {, }
$$

and on the other hand, Darcy's law states that

$$
\vec{v}=-\operatorname{grad}(p) \text {. }
$$

Combining (1.1) and (1.2) we obtain

$$
u_{t}=\operatorname{div}(u \operatorname{grad}(p))
$$

The usual assumption is now that the pressure is proportional to a power of the density, for example $p=u^{m-1}$, with $m>1$. Substituting this in (1.3) we get, up to a multiplicative constant, $u_{t}=\Delta u^{m}$. Taking into consideration the initial distribution of the gas and the boundary conditions, we are led to consider the

Received by the editors January 4, 1993.

1991 Mathematics Subject Classification. Primary 35K60, 35K65.

Key words and phrases. Diffusion, porous media.

The authors were partially supported by FONDECYT under Grant 91-0813. 
following problem as a model for the flow of gas in a porous container:

$$
\begin{aligned}
u_{t} & =\Delta\left(u^{m}\right) \text { in } D \times[0,+\infty), \\
u(x, 0) & =u_{0}(x) \text { on } D, \\
a u(x, t)+b \frac{\partial u^{m}}{\partial \vec{n}}(x, t) & =h(x, t) \text { on } \partial D \times[0,+\infty),
\end{aligned}
$$

where $D \subset R^{N}$ is a domain with smooth boundary.

For a general review on the above-stated and related problems we refer to [A].

One important feature of the solutions, actually weak solutions, of this problem is that perturbations propagate with a finite speed. By this we understand that if $z \in$ int $D$ and $z$ is exterior to the support of $u_{0}$, then there exists a time $T(z)$ so that $u(z, t) \equiv 0 \quad \forall t<T(z)$ and $u(z, t)>0$ for $t>T(z)$. See [CF] for the case of the Cauchy problem in $R^{N}$. The time $T(z)$ represents the time it takes the gas to reach the point $z$.

The aim of this note is to give an estimate for the time $T(z)$ in terms of the initial and the boundary data. In the case of the Cauchy problem in $R^{N}$ the Harnack's type inequality that appears in $[\mathrm{AC}]$ gives not only an estimate for $T(z)$ but also a lower bound for the density $u(z, t)$ in terms of the initial data. The last-mentioned inequality has been used in [CVW] to study the free boundary of solutions of (1.4) for the Cauchy problem in $R^{N}$.

\section{The NeumanN PROBLEM IN BOUNDed CONVEX Domains}

In this section we assume that $D$ is a bounded convex domain in $R^{N}$ with smooth boundary and that $a=0$ and $b=1$ in (1.6). Moreover, we suppose that the initial condition $u_{0}$ and the boundary data $h$ are nonnegative smooth functions. Under these hypotheses it is a consequence of the maximum principle that the solution $u(x, t)$ is nonnegative.

For any $z \in D$ consider the function $W(x, z)=1 /\|z-x\|^{N}$ and define

$$
R(t)=\int_{D} u(y, t) W(y, z) d y .
$$

We assume that $z$ does not belong to the support of $u_{0}$. We note that $R(t)$ is finite as long as $t<T(z)$ and it becomes $+\infty$ for $t>T(z)$. Therefore, we can estimate $T(z)$ by estimating the blow-up time for the quantity $R(t)$.

In what follows all the calculations we will make can be justified as long as $z$ does not belong to the support of $u(\cdot, t)$.

The usual computations using Stokes's Theorem give

$$
\begin{aligned}
\frac{d R}{d t}= & \int_{D} u^{m}(y, t) \Delta W(y, z) d y \\
& +\int_{\partial D} \frac{\partial u^{m}(y, t)}{\partial \vec{n}} W(y, z) d y-\int_{\partial D} u^{m}(y, t) \frac{\partial W}{\partial \vec{n}}(y, z) d y .
\end{aligned}
$$

Since $D$ is convex, the exterior normal derivative of $W$ is nonpositive, so the last term on the right of (2.2) can be dropped to obtain

$$
\frac{d R}{d t} \geq \int_{D} u^{m}(y, t) \Delta W(y, z) d y+\int_{\partial D} \frac{\partial u^{m}(y, t)}{\partial \vec{n}} W(y, z) d y .
$$


We observe now that as a consequence of Holder's inequality we have

$$
\int_{D} u^{m}(y, t) \Delta W(y, z) d y \geq \frac{\left(\int_{D} u(y, t) W(y, t) d y\right)^{m}}{\left(\int_{D}\left(\frac{W^{m}}{\Delta W}\right)^{1 /(m-1)}(y, t) d y\right)^{m-1}} .
$$

Substituting (2.4) into (2.3) and recalling that $W(y, z)=1 /\|y-z\|^{N}$, we obtain

$$
\frac{d R}{d t} \geq C(z) R^{m}(t)+\int_{\partial D} h(y, z) W(y, z) d y
$$

where

$$
C(z)=\frac{2 N}{\left(\int_{D}\|z-y\|^{(2-N(m-1)) /(m-1)}\right)^{m-1}} .
$$

We can get now the desired estimate from (2.5) as follows.

Fix $t_{0}<T(z)$. Dropping the first term in the right-hand side of (2.5) and integrating from 0 to $t_{0}$ we get

$$
R\left(t_{0}\right) \geq \int_{0}^{t_{0}} \int_{\partial D} h(y, t) W(y, z) d y d t+R(0) .
$$

Dropping the second term in the right-hand side of (2.5) and integrating from $t_{0}$ to $t$ we obtain

$$
R^{m-1}(t) \geq \frac{R^{m-1}\left(t_{0}\right)}{1-C(z)(m-1) R^{m-1}\left(t_{0}\right)\left(t-t_{0}\right)}
$$

Now (2.7) and (2.6) provide the following estimate for $T(z)$ :

$$
T(z) \leq t_{0}+\frac{1}{2 N(m-1)}\left(\frac{\int_{D}\|z-y\|^{(2-N(m-1)) /(m-1)} d y}{\int_{0}^{t_{0}} \int_{\partial D} \frac{h(y, t)}{\|y-z\|^{N}} d y d t+R(0)}\right)^{m-1}
$$

for any $0<t_{0}<T(z)$.

In order to give an idea about the meaning of (2.8) we consider the special case when the Neumann boundary data is independent of time, that is, $h(x, t)=h(x)$, and the initial condition vanishes. With these assumptions, taking $t_{0}=\lambda T(z)$ with $0<\lambda<1$ in (2.8), we get

$$
(T(z))^{m} \leq \frac{1}{(1-\lambda) \lambda^{m-1}} \frac{1}{2 N(m-1)}\left(\frac{\int_{D}\|z-y\|^{(2-N(m-1)) /(m-1)} d y}{\int_{\partial D} \frac{h(y)}{\|y-z\|^{N}} d y}\right)^{m-1} .
$$

Minimizing the quantity $\frac{1}{(1-\lambda) \lambda^{m-1}}$ for $0<\lambda<1$ we obtain the estimate

$$
(T(z))^{m} \leq \frac{1}{2 N}\left(\frac{m}{m-1}\right)^{m}\left(\frac{\int_{D}\|z-y\|^{(2-N(m-1)) /(m-1)} d y}{\int_{\partial D} \frac{h(y)}{\|y-z\|^{N}} d y}\right)^{m-1}
$$

We finally observe that the numerator in (2.10) measures the shape of the domain near the point $z$ in the sense that if the domain is narrow near $z$, the numerator is small. On the other hand the denominator measures the distance from $z$ to the source $h$. 


\section{The NeumanN PRoblem IN THE half SPACE}

In this section we sketch the idea of how to deal with the case when $D$ is the half space $D=\left\{\left(x_{1}, x_{2}, \ldots, x_{N}\right) / x_{N} \geq 0\right\}$.

All the arguments of the previous section can be carried over in this case, with the only exception of inequality (2.4) because the denominator, in this case, becomes infinite.

In order to fill this gap we recall that solutions of (1.4) satisfy the following conservation law:

$$
\int_{D} u(y, t) d y=\int_{0}^{t} \int_{\partial D} h(y, s) d y d s+\int_{D} u_{0}(y) d y \equiv H(t) .
$$

Now, to obtain a substitute for (2.4), we consider the problem of minimizing the functional

$$
J(w)=\int_{D} w^{m}(y) \Delta W(y, z) d y
$$

subject to the conditions

$$
w \geq 0, \quad \int_{D} w(y) W(y, z) d y=R, \quad \text { and } \quad \int_{D} w(y) d y=H .
$$

This problem can be solved by using elementary techniques of calculus of variations. In doing so we get that there exists a positive constant $C(m, N)$ such that the minimum value $\bar{M}$ of $J(w)$ subject to the above stated conditions satisfies

$$
\bar{M} \geq C(m, N) \frac{R^{m+2 / N}}{H^{2 / N}} .
$$

Moreover, the constant $C(m, N)$ is given by

$$
C(m, N)=\min _{0 \leq \alpha \leq 1}\left(I_{1}^{2 / N}(\alpha) / I_{2}^{m+2 / N}(\alpha)\right)\left(I_{2}(\alpha)-I_{1}(\alpha)\right)
$$

where

$$
I_{1}(\alpha)=\int_{\left\{\left(x_{1}, \ldots, x_{N}\right) / x_{N} \geq-\alpha\right\}}\left(\left(\|x\|^{2}-\|x\|^{N+2}\right)_{+}\right)^{1 /(m-1)} d x
$$

and

$$
I_{2}(\alpha)=\int_{\left\{\left(x_{1}, \ldots, x_{N}\right) / x_{N} \geq-\alpha\right\}}\left(\left(\|x\|^{2}-\|x\|^{N+2}\right)_{+}\right)^{1 /(m-1)} \frac{d x}{\|x\|^{N}} .
$$

Therefore, using the definition of $R(t)$ and (3.1), we have

$$
\int_{D} u^{m}(y, t) \Delta W(y, z) d y \geq C(m, N) \frac{R^{m+(2 / N)}(t)}{H^{2 / N}(t)} .
$$

Substituting (3.2) in (2.3) and repeating the integration argument of the preceeding section we get

$$
\begin{aligned}
& \int_{t_{0}}^{T(z)} \frac{d s}{\left(\int_{D} u_{0}(y) d y+\int_{0}^{s} \int_{\partial D} h(y, r) d y d r\right)^{2 / N}} \\
& \quad \leq \frac{m+2 / N-1}{C(m, N)} \frac{1}{\left(R(0)+\int_{0}^{t_{0}} \int_{\partial D} \frac{h(y, s)}{\|y-z\|^{N}} d y d s\right)^{m+(2 / N)-1}}
\end{aligned}
$$

for any $0<t_{0}<T(z)$, which is our estimate for $T(z)$ in this case. 
We note that, in the special case that $u_{0} \equiv 0$ and $h(x, t) \equiv h(x)$, the same arguments of the previous section allow us to get the estimate

$$
(T(z))^{m} \leq K(m, N) \frac{\left(\int_{\partial D} h(y) d y\right)^{2 / N}}{\left(\int_{\partial D} \frac{h(y)}{\|y-z\|^{N}} d y\right)^{m+(2 / N)-1}},
$$

where $K(m, N)$ is a constant that depends on $m$ and $N$ only.

We would like to finish this note with the observation that, perhaps, using a different kernel $W(x, z)$ one could estimate the time $T(z)$ in the case of more general domains with other type of boundary conditions.

\section{ACKNOWLEDGMENT}

The authors would like to thank the referees for several useful comments.

\section{REFERENCES}

[A] D. G. Aronson, Nonlinear diffusion problems, Free Boundary Problems: Theory and Applications (A. Fasano and M. Primicerio, eds.), Pitman, New York, 1983, pp. 135-149.

[AC] D. G. Aronson and L. A. Caffarelli, The initial trace of the solution of the porous medium equation, Trans. Amer. Math. Soc. 280 (1983), 351-366.

[CF] L. A. Caffarelli and A. Friedman, Regularity of the free boundary of a gas flow in an n-dimensional porous medium, Indiana. Univ. Math. J. 29 (1980), 361-391.

[CVW] L. A. Caffarelli, J. L. Vazquez, and N. I. Wolanski, Lipschitz continuity of solutions and interfaces of the $N$-dimensional porous medium equation, Indiana Univ. Math. J. 36 (1987), 373-401.

Facultad de Matemáticas, Universidad Católica, Casilla 306, Santiago, Chile

E-mail address: melgueta@mat.puc.cl 